\title{
In ovo exposure of Eurycoma longifolia extract on the pre-hatched development of chicken embryo
}

\author{
Rahman MS ${ }^{1 *}$, LC Yuen, NN Rasyidah Nik Hasan
}

Faculty of Sustainable Agriculture, Universiti Malaysia Sabah (Sandakan Campus), Locked Bag No. 03, 90509 Sandakan, Sabah, Malaysia; ${ }^{1}$ Department of Poultry Science, Bangladesh Agricultural University, Mymensingh2202, Bangladesh

\begin{abstract}
This study was conducted to investigate the effects of in ovo administration of Eurycoma longifolia (Tongkat Ali) extract (TAE) on the survivability and pre hatch development of chicken embryo. A dose response study of $0.1 \mathrm{mg}, 1 \mathrm{mg}$ and $10 \mathrm{mg} \mathrm{TAE} / 100 \mathrm{~g}$ egg on embryo survivability during first 5 days of incubation showed that the 0.1 to $1 \mathrm{mg}$ exposures had no effect on embryo survivability, and the lethal dose to kill $50 \%\left(L D_{50}\right)$ was at around $10 \mathrm{mg}$. The $0.1 \mathrm{mg}$ exposure was found to be non-lethal to the developing embryo until $19^{\text {th }}$ day of incubation and it also enhanced $(p<0.05)$ the somite development (42 in TAE and 38 in control embryos) in 88 to 96 hours embryo. Then $0.1 \mathrm{mg}$ TAE exposure resulted in $70.5 \%$ body mass gain ( $\%$ to egg weight) in the $19^{\text {th }}$ day embryo in contrast to $53.4 \%$ in the control. Compared to control, significant increases in limb lengths and feather-follicles in 9-day old embryos were observed in the $0.1 \mathrm{mg}$ TAE group. On $19^{\text {th }}$ day of incubation, the $100 \%$ of the $0.1 \mathrm{mg}$ TAE exposed embryos were found to produce traceable vocal sounds along with the pipping marks on egg shell, but these phenomena were almost absent in the control chicks during the same period of incubation. Results suggested that the TAE exposure at $>1 \mathrm{mg} / 100 \mathrm{egg}$ dose is lethal to developing chicken embryo, but the $0.1 \mathrm{mg} \mathrm{TAE} / 100 \mathrm{~g}$ egg exposure through in ovo injection can be a beneficial strategy to produce heavier and stronger day old chicks.
\end{abstract}

Key words: chick embryo, embryo mortality, embryonic development, in ovo feeding Bangladesh Animal Husbandry Association. All rights reserved. $\quad$ Bang. J. Anim. Sci. 2016. 45 (2): 24-29

\section{Introduction}

The administration of nutrients into hatching eggs is called in ovo feeding (Uni et al., 2005). In ovo feeding is able to affect the performance such as digestion capacity, immunity, growth and feed efficiency of hatched out chicks by providing feed to the developing embryo through amniotic fluid. According to Pal et al. (2002), slight amendment in nutritional technology during embryonic development is significant, because any changes during this period can affect embryonic metabolism with consequent impact on body composition and organ development of the chicks through changes in the nutrient utilizations pattern. The study of embryonic developmental biology or pre hatch development of embryo in chicken is not only important for poultry industry but also as a model to study the effects of specific nutritional supplements for humans, because chicken's embryo development information can easily be extrapolated in human case (Gelder and VanBelanger, 1989).

*Corresponding author: msrahman@ums.edu.my
The improvement in body weight gain of poultry species during last two decades has successfully been made through the pre-hatch development (Hulet, 2007). In the current era of poultry nutrition, scientists have paid strong attention to early feeding of chicken to boost their pre hatch health and immunity (Ferket and Uni, 2011). Consequently, scientists are exploring varieties of substances as early feed ingredients in poultry. The substances range from purified nutrients to enzymes or synthetic hormones. The responses of many substances on the early development of poultry species are already well documented (Moran, 2007; Gender and VanBelanger, 1989).

Eurycoma longifolia Jack, commercially known as Tongkat Ali is a famous medicinal plant in the family of Simaroubaceae (Effendy et al., 2012). It has been widely distributed in South East Asia including Malaysia, Vietnam, Indonesia and Thailand. Tongkat Ali root extract (TAE) reserves a greater range of medicinal property for general health enhancer as well as for the improvement 
of overall quality of life. Nowadays, TAE can be found in varieties of dietary supplements with intention to improve the libido, energy levels as well as restorer of testosterone levels in human. TAE contains a wide range of chemicals such as eurycomanone, eurycomanol, eurycomalactone, canthine-6-one alkaloid, 9-hydroxycanthin-6one, $14,15 \beta$ dihydroxyklaineanone, phenolic components, tannins, quanissoids, and triterpenes. The presence of these chemicals enables TAE to possess effective medicinal value in sexual enhancement, antimalarial, antibacterial as well as antitumor. TAE has been well documented to exert anti oxidative properties due to its high concentrations of superoxide dismutase (SOD). It is famously known for its aphrodisiac effect (Effendy et al., 2012). In experimental condition, TAE feeding on numerous rodents have been found to restore the testosterone levels also (Talbott et al., 2013). The information on the effect of TAE exposure on chicken embryo development is still lacking, especially through in ovo exposure method. Therefore, this study was designed to investigate the efficacy of TAE as in ovo feed additive on embryonic mortality, weight gain and body parts development at pre hatch period.

\section{Materials and Methods}

This study was conducted at the Faculty of Sustainable Agriculture (FSA), Universiti Malaysia Sabah in Malaysia. A total of 160 fertile eggs ranging from 50 to $70 \mathrm{~g}$ weight from cross bred chicken were used in this experiment. Powdered Tongkat Ali root extract (TAE) were used as the test chemical. Each dose of TAE was dissolved in a fixed volume of $0.5 \mathrm{ml}$ of sterile saline. Initially, a dose response study of TAE with $0 \mathrm{mg}, 0.1 \mathrm{mg}$, $1 \mathrm{mg}$ and $10 \mathrm{mg}$ of TAE/ $100 \mathrm{~g}$ egg was conducted on 60 fertile eggs keeping 15 eggs under each dose. The doses were evaluated for their lethal effects on developing embryo during first 5 days of incubation. Then, the $0.1 \mathrm{mg} T A E / 100$ egg dose was chosen as the appropriate dose for further study because of its lower lethality to the embryo. Later on, this $0.1 \mathrm{mg}$ TAE $/ 100 \mathrm{~g}$ egg was used to investigate the effects of TAE on survivability, morphological appearance and body parts development of the embryo until $19^{\text {th }}$ day of incubation against control group. The TAE solution was injected (with 25 gauge needle) into the middle air sac of the fertile eggs before placing them into force-draft and auto turning electronic incubator (Brinsea-250, UK). After injection, the hole on the egg shell was sealed with a piece of mending tape. All eggs were marked separately before placing them on the setting trays of the incubator. The incubator was set at $38.5^{\circ} \mathrm{C}$ with $70 \%$ relative humidity having auto turning at hourly interval. The morphological and body parts development were monitored at 88-92 hours, 9th day, 16th day and 19th day of incubation. Each time, 10 eggs under a dose were broken and open out to measure the desired embryonic parameters.

The morphological development of the embryo was compared with the standard development pattern of the chicken embryo as stated by Hamburger and Hamilton (1951). The somite developments at 88 to 92 hours of incubation was studied using compound microscope, whereas the embryo weight was measured by electronic balance on 9th, 16th and 19th day of incubation. The wing, limb and whole body length of the developing chick embryo were measured on the 9th day of incubation and onwards. The beak length and third toe length were measured at 16 th and 19th dayof incubation only, because the development of these body parts is dominant at this time phase. The embryonic death was counted on total incubation period as well as on $0-5,6-9$ and $10-19^{\text {th }}$ day of incubation. The vocal sound of the developed embryo was traced at $19^{\text {th }}$ day of incubation. The collected data was analysed by T-tests and Chi-square test using Statistical Package for Social Science (SPSS) version 22. In each case, the significant difference was considered at $p<0.05$.

\section{Results and Discussion}

Figure 1 shows the dose responses of TAE on chicken embryonic mortality during first 5 days of incubation. It was noticed that TAE exposure to developing chicken embryo up to $1 \mathrm{mg} / 100 \mathrm{~g}$ egg is not lethal (Figure 1. A). However, the $L_{50}$ has been detected at around $10 \mathrm{mg} / 100 \mathrm{~g}$ egg dose of TAE. Figure $1(B)$ indicated that $0.1 \mathrm{mgTAE} / 100 \mathrm{~g}$ egg is safer to the developing embryo until $19^{\text {th }}$ day of incubation also. Developmental phase-wise distribution of the embryonic mortality shown in Figure 2 indicated that $0.1 \mathrm{mg}$ TAE and control 


\section{Tongkat Ali exposure on chicken embryo development}

chicks have similar pattern of embryonic mortality until $19^{\text {th }}$ day of incubation.

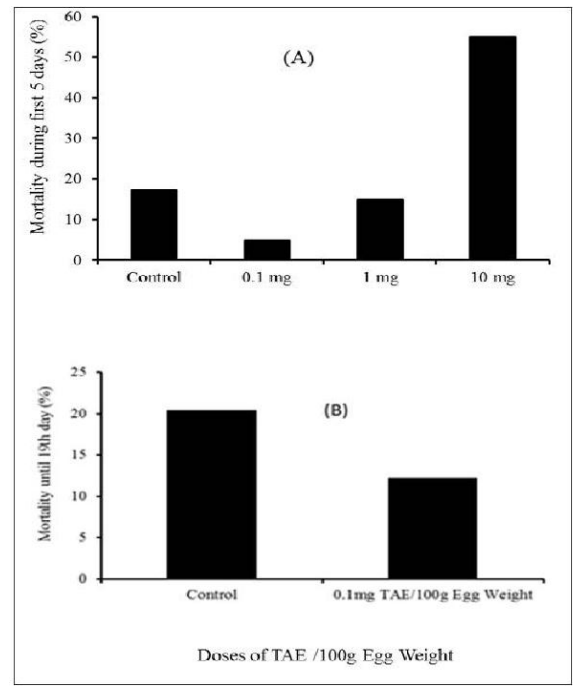

Figure 1. Effects of in ovo Tongkat Ali Extract (TAE) exposure on the survivability of developing chicken embryo during first 5 days (A) and 19 days (B) of incubation. Results taken from the observation on 60 eggs $(\mathrm{A})$ and 100 eggs (B) those had equally been distributed to each TAE dose. In figure $(A)$, the value in $10 \mathrm{mg}$ was significantly different $(p<0.05)$ from that of control under Chi-square test.

Actually, eurycomanone, the main component identified in the n-butanol fraction of TAE is the most toxic constituent of Eurycoma longifolia (Chan and Choo, 2002). They reported a LD $D_{50}$ of this compound in rat isaround $0.05 \mathrm{~g} / \mathrm{kg}$ body weight. The $\mathrm{LD}_{50}$ at $10 \mathrm{mg} / 100 \mathrm{~g}$ egg found in our study can be explained by the same reason. In fact, our $\mathrm{LD}_{50}$ dose in chicken was almost double than that in the rat (Chan and Choo, 2002). The $\mathrm{LD}_{50}$ for oral administration of aqueous TAE on rat was more than $5 \mathrm{~g} / \mathrm{kg}$ body weight and it was unlikely to cause any fatality in human whose equivalent dose of TAE is $35 \mathrm{~g}$ per $70 \mathrm{~kg}$ body weight (Shuid et al., 2011). The difference in $L D_{50}$ dose for different species could be explained by individual variation and variation in the route of exposures also.

The in ovo exposure is an indirect method of exposure to the developing embryo in contrast to direct intramuscular or intravenous injection in rat. However, it can be assumed that the appropriate doses of TAE are crucial to have optimum effects in chicken embryonic model. The observation on embryonic somite development at
88 to 92 hours of incubation (Photo 1 ) showed a significant difference $(p<0.005)$ between control (38 somites) and TAE (42 somites) exposed eggs.
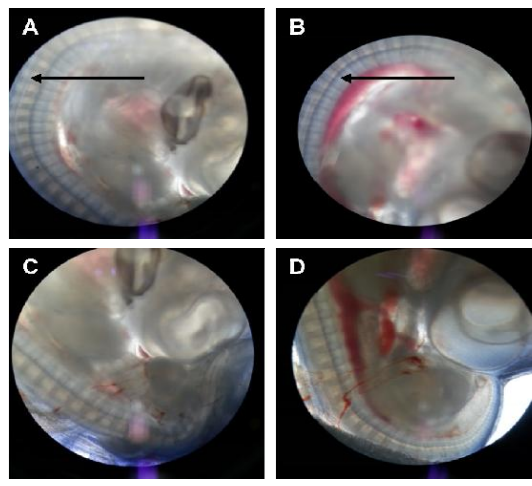

Photo 1. Representative photos of the embryonic somite development in in ovo Tongkat Ali root extract (TAE) injected and control chicken embryos; $\mathrm{A}$ and $\mathrm{C}$ are the head and tail parts of the in ovo saline injected embryo, and $B$ and $D$ are the head and tail parts of the in ovo TAE injected embryos. Black arrow indicates somite development in the chicken embryo at 88 to 92 hours of incubation.

The effect of in ovo TAE exposure on embryonic weight has been shown in Table 1. It was noticed that the TAE exposure did not affect the embryonic weight gain on $9^{\text {th }}$ and 16 th day of incubation, but it resulted $17.12 \%$ higher $(p>0.05)$ body mass gain in the live embryos compared to that in the control.

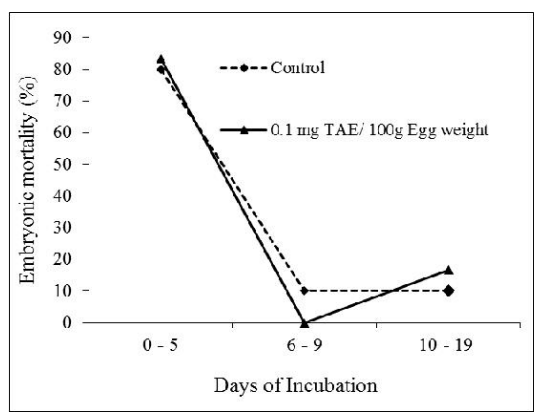

Figure 2. Distribution of the in-ovo Tongkat Ali root extract (TAE) exposed embryonic mortality in chicken at different time periods of incubation. Observations were made on total 100 eggs.

Our finding was in agreement with Coutinho et al. (1993), who apprehended that higher embryonic weight is closely related to somites development. Schmidt et al. (2003) also reported a relationship between somites development during 40 to 50 hours of incubation and the rate of cell division. $\mathrm{He}$ also established a relationship in the carcass 
and breast yield in birds with somite formation and development.

Table 1. Effect of in ovo exposure of Tongkat Ali root extract (TAE) on weight gain in chicken embryo (\% to egg weight) during 9th until 19th day of incubation

\begin{tabular}{llll}
\hline Day of & \multicolumn{2}{c}{ Treatment } & Significance \\
\cline { 2 - 3 } Incubation & Control & TAE & \\
$9^{\text {th }}$ day & 7.81 & 7.37 & NS \\
$16^{\text {th }}$ day & 45.08 & 45.03 & NS \\
$19^{\text {th }}$ day & 53.38 & 70.50 & NS \\
\hline
\end{tabular}

NS, $p>0.05$ when compared with control in the same row. Measurement on a specific period was taken from at least 7 embryos under each treatment group.

The $17.12 \%$ higher embryonic weight at $0.1 \mathrm{mg}$ $\mathrm{TAE} / 100 \mathrm{~g}$ egg exposure during $19^{\text {th }}$ day of incubation might be the result of TAE's growth enhancing effects, because TAE is a known testosterone booster which can increase testosterone levels up to $440 \%$ and testosterone is responsible for increasing metabolic rates, oxidative phosphorylation and c-GMP production. Andersson et al. (2004) also speculated a weaker immune function at highly testosterone exposed immune system, because in testosterone exposed organism most of the metabolic energy is allocated for the growth.
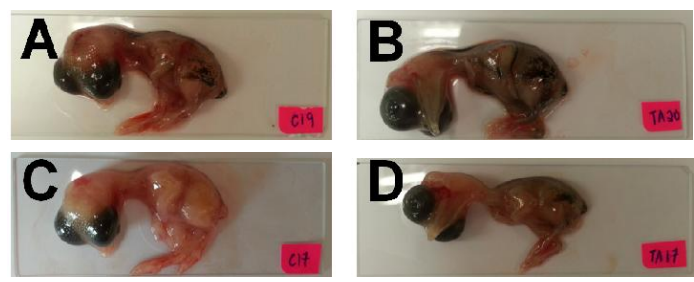

Photo 2. Representative photos of the embryonic feather follicle development in in ovo Tongkat Ali extract (TAE) injected and control chicken embryo at $9^{\text {th }}$ day of incubation; A, control embryo showing the development of feather germs on wing, thigh and midventral line, $B, T A E$ injected embryo showing the development of feather germs on wing, thigh, midventral line, tail and other parts of body, C, Control embryo showing no development of feather germs, D, TAE injected embryo showing the development of feather germs on wing, thigh, mid-ventral line and tail. The black arrow indicated the presence of featherfollicles on the chick embryo and red tag was the embryo tag number.

On the other hand, Uller and Olsson (2003) reported a conversion of excess testosterone to other steroids resulting weaker response to growth during embryonic development what contradicted our current findings. However, the response of steroids to growth and development might be varied in the organism depending on their stages of growth and sex also (Rahmanet al, 2009).TAE exposure (Photo 2) created a distinct development of feather germs on the wing, thigh, mid ventral line and tail of $100 \%$ chick embryos. On the other hand, in control 33\% was with no development, $33 \%$ was with visible development on the thigh, wing and mid ventral line, $17 \%$ was with the development on limb and mid-ventral line and $17 \%$ was with feather follicles development on mid-ventral line in $9^{\text {th }}$ day embryo. To the best of our knowledge, there is still a lacking of information to support this study finding. According to Hamburger and Hamilton (1951), feather-germs on chick embryo started to grow at around 6.5th day. Therefore, our current findings on vigorous feather follicle development along with higher body mass in the TAE exposed embryos promoted us to apprehend that featherfollicles development is an indication for faster embryonic growth and development during incubation.

Table 2. Effect of in ovo Tongkat Ali root extract (TAE) exposure on limb length (\% to embryo length) of chicken embryo during 9 th until 19 th day of incubation

\begin{tabular}{llll}
\hline \multirow{2}{*}{$\begin{array}{l}\text { Day of } \\
\text { Incubation }\end{array}$} & \multicolumn{2}{c}{ Treatment } & Significance \\
\cline { 2 - 3 } & Control & TAE & \\
\hline 9th day & 37.50 & 39.93 & NS \\
16th day & 62.72 & 68.63 & NS \\
19th day & 52.10 & 68.10 & * \\
\hline
\end{tabular}

${ }^{*}, p<0.05$; NS, $p>0.05$ when compared with control in the same row. Measurement on a specific period was made on at least 7 embryos under a treatment group.

In the present study, we did not find any effect of TAE exposure on wing and third toe development those are also good indicator of growth and development in embryo. However, we observed that TAE treated $100 \%$ embryo produced a traceable vocal sound and showed pipping signs on the eggshell; whereas both of the symptoms were completely absent in the control embryos. Actually, vocal sound production and pipping is a part of the synchronization of hatching process in avian species. Several studies have mentioned that hormone has roles on parental control over the phenotypes of the offspring (Groothuis et al., 


\section{Tongkat Ali exposure on chicken embryo development}

2005; Smiseth et al., 2011). This finding was further supported by Irving et al. (1976), who described the role of testosterone hormone to stimulate the production of red blood cells in cultured chick blastoderm that facilitated rapid growth and synchronization in hatching process.
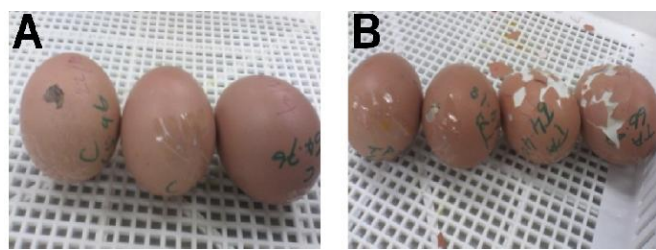

Photo 3. A representative photo of the pipping signs/chick's body stretching impact on the eggshell of the control $A$ and in ovo TAE injected groups, $B$ at $19^{\text {th }}$ day of incubation

The above results and discussion lead us to conclude that $0.1 \mathrm{mg}$ TAE exposure per $100 \mathrm{~g}$ egg through in ovo injection had very clear effects on chicken embryonic development, possibly through endocrine channels. The TAE exposure at $>1 \mathrm{mg} /$ 100 egg was lethal to the developing chicken embryo, but the $0.1 \mathrm{mg}$ TAE/ $100 \mathrm{~g}$ egg exposure through in ovo injection might be a beneficial strategy to produce the heavier and stronger embryo followed by earlier hatch out of the chicks. But, further studies on the mechanisms of TAE actions are recommended to strength the current findings.

\section{Acknowledgements}

The financial support of this research came from Universiti Malaysia Sabah (UMS) research grant no. SBK0119-STWN-2014. The Tongkat Ali (Eurycoma longifolia) extract was donated by Mr. Awang Ahmad Bin Mohd Yunus, Senior Lecturer, Department of International Tropical Forestry, UMS, Malaysia.

\section{References}

Andersson S, Uller T, Lohmus M. and Sunstrom F (2004). Effects of egg yolk testosterone on growth and immunity in a precocial bird. Journal of Evolutionary Biology. 17(3): 501-505.

ChanKL and Choo CY (2002). The toxicity of some quassinoids from Eurycoma longifolia. Planta Medica. 68(7): 662-664.

Coutinho LL, Morris J, Marks HL, BuhrJR and Ivarie R (1993). Delayed somite formation in a quail line exhibiting myofiber hyperplasia is accompanied by delayed expression of myogenic regulatory factors and myosin heavy chain. Development. 17: 563-569.

Effendy NM, Mohamed N, Muhammad N, Mohamad IN and Shuid AN (2012). Eurycoma longifolia: medicinal plant in the prevention and treatment of male osteoporosis due to androgen deficiency. Evidence Based Complementary and Alternative Medicine. 125761.

Ferket PR and Uni Z (2011). The egg comes first: inovo feeding and the promise of perinatal nutrition. In: Alltech Animal Health and Nutrition Symposium, Kentucky (USA).

Gelder NM and VanBelanger FS (1989). Nutrition and selective uptake of free amino acid necessary for the development of the brain of the chicken embryo. Arch Internationales de Physiologie et de Biochimie. 94: A265-A266.

Groothuis TG, Muller W, VonEngelhardt N, Carere C and Eising CM (2005). Maternal hormones as a tool to adjust offspring phenotype in avian species. Neurosceince and Biobehaviour Review. 29: 329-352.

Hamburger $\mathrm{V}$ and Hamilton HL (1951). A series of normal stages in the development of the chick embryo. Developmental Dynamic. 195(4): 231272.

Hulet RM (2007). Managing incubation: where are we and why? Poultry Science. 86:1017-1019.

Irving RA, Mainwaring WIP and Spooner PM (1976). Regulation of hemoglobin synthesis in cultured chick blastoderms by steroids related $\mathrm{t} 5$-betaandrostane. The Journal of Biochemistry. 154: 81-93.

Moran ET (2007). Nutrition of the developing embryo and hatchling. Poultry Science. 86 (5): 10431049.

Pal AK, Quadri MA, Jadhao SB, Kumbhaka J, Kushwah HS and Datta IC (2002). genotype influences body composition of developing chicken embryo. Pakistan Journal of Nutrition. 1(2): 82- 84

Rahman MS, Mochizuki M and Mori M (2009). Inovo cadmium toxicity in developing quail embryo (Coturnix japonica): sex-dependent responses to ascorbic acid protection. The Journal of Poultry Science. 46 (4): 334-39.

Schmidt GS, Coutinho LL, Figuiredo EAP, Ledur MC and Alves HJ (2003). Morphological genetic markers for selection of broiler lines.Brazilian Journal of Poultry Science. 5(3): 175-178.

Shuid AN, Siang LK, Chin TG, Muhammad N, Soelaiman IN (2011). Acute and subacute toxicity studies of Eurycoma longifolia in male rats. International Journal of Pharmacology. (7): 641- 646. 
Rahman et al. (2016) Bang. J. Anim. Sci. 45 (2): 24-29

Smiseth PT, Pellissier SM and Andews C (2011). Hormonal regulation in offspring begging and mediation of parent-offspring conflict.Animal Behaviour. 81: 501-517.

Talbott SM, Talbott JA, George A and Pugh M(2013). Effect of tongkat ali on stress hormone and psychological mood state in moderately stressed subjects. Journal of International Society of Sport Nutrition. 10: 28.
Uller T and Olsson M(2003). Prenatal exposure to testosterone increases ectoparasite susceptibility in the common lizard (Lacerta vivipara). Proceedings of the Royal Society of London Series. B 270: 1867-1870.

Uni Z, Ferket PR, Tako E and Kedar O (2005). In ovofeeding improves energy status of late-term chicken embryos. Poultry Science. 84: 764-770. 